\title{
Measurement of viral load by the automated Abbott real-time HIV-1 assay using dried blood spots collected and processed in Malawi and Mozambique
}

\author{
F Erba, ${ }^{1}$ MSc; D Brambilla, ${ }^{2}$ MSc; S Ceffa, ${ }^{2}$ MSc; F Ciccacci,${ }^{2,3}$ MD; R Luhanga,${ }^{4}$ BSc; Z Sidumo, ${ }^{5}$ BSc; L Palombi, ${ }^{6}$ MD; \\ S Mancinelli, ${ }^{6} \mathrm{MD}$; M C Marazzi, ${ }^{7} \mathrm{MD}$; M Andreotti, ${ }^{8} \mathrm{MSc} ;$ M Giuliano, ${ }^{8} \mathrm{MD}$ \\ ${ }^{1}$ Department of Clinical Science and Translational Medicine, University of Roma Tor Vergata, Rome, Italy \\ ${ }^{2}$ DREAM Programme, Community of Sant'Egidio, Rome, Italy \\ ${ }^{3}$ Department of Infectious Diseases, University of La Sapienza, Rome, Italy \\ ${ }^{4}$ DREAM Programme, Community of Sant'Egidio, DREAM Health Centre, Blantyre, Malawi \\ ${ }^{5}$ DREAM Programme, Community of Sant'Egidio, Centro Para Criança, Maputo, Mozambique \\ ${ }^{6}$ Department of Biomedicine and Prevention, University of Roma Tor Vergata, Rome, Italy \\ ${ }^{7}$ Department of Community Health, LUMSA University, Rome, Italy \\ ${ }^{8}$ Department of Therapeutic Research and Medicines Evaluation, Istituto Superiore di Sanità, Rome, Italy
}

Corresponding author: $M$ Andreotti (mauro.andreotti@iss.it)

Background. The use of dried blood spots (DBS) for HIV-1 viral load quantification can greatly improve access to viral monitoring for HIV-infected patients receiving treatment in resource-limited settings.

Objectives. To evaluate and validate HIV viral load measurement from DBS in sub-Saharan Africa, with a reliable, all-automated, standard commercial assay such as the Abbott m2000.

Methods. A total of 277 DBS were collected in different health centres in Malawi and Mozambique and analysed for viral load determination using the Abbott m2000 assay with the corresponding plasma samples as gold standard. Samples were extracted using the m2000SP automatic extractor and then processed as the plasma samples using the specific 1.0 mL HIV-RNA DBS protocol.

Results. Among samples with detectable HIV-RNA the correlation between viral load obtained from the paired 131 plasma and DBS samples was high $(r=0.946)$. Overall, viral load values between DBS and plasma differed by less than $0.5 \log$ unit in $90.1 \%$ of cases and by less than $1 \log$ unit in $100 \%$ of cases. Using a threshold of 1000 copies $/ \mathrm{mL}$ (defining virological failure in resource-limited settings), sensitivity was $94.2 \%$ and specificity $98.6 \%$, and both positive and negative predictive values were high ( $98.5 \%$ and $94.5 \%$, respectively).

Conclusion. DBS extracted and processed using the Abbott automated system can be reliably used in resource-limited setting to diagnose virological failure.

S Afr Med J 2015;105(12):1036-1038. DOI: 10.7196/SAMJ.2015.v105i12.9673

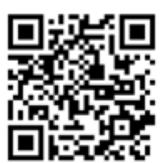

The use of dried blood spots (DBS) has greatly facilitated genetic and metabolic screening of newborns by simplifying sample collection, storage and transport. Measuring the HIV-1 burden from whole blood dried on filter paper provides a suitable alternative for lowtechnology settings with limited access to laboratory facilities, such as subSaharan Africa (SSA). Nucleic acids in DBS have been shown to be stable for several months at room temperature, provided the DBS specimens are thoroughly dried and are stored with desiccant. ${ }^{[1-4]}$ DBS specimens can therefore be collected at remote rural sites and transported to a central or regional testing laboratory without refrigeration. DBS are user-friendly and cost-effective in resource-limited settings, their ability being demonstrated to correctly identify virological failure. ${ }^{[5-7]}$ DBS use may therefore represent a paradigm shift in accessibility to virological testing for HIV infection. Indeed, the Diagnostic Access Initiative (http://www.unaids.org) signed in Melbourne during the 20th International AIDS Conference in July 2014, specifically focused on ensuring that all people accessing HIV treatment should have ready access to tests that monitor levels of the virus in their bodies, and underlined the importance of developing new and affordable viral load and early infant diagnosis technology.

The objective of this study was to evaluate and validate, in SSA, HIV viral load measurement from DBS with a reliable, all-automated, standard commercial assay, the Abbott m2000 (Abbott Molecular, USA). The study was performed in two healthcare settings in two different countries,
Malawi and Mozambique, within the health centre network of the DREAM (Drug Resource Enhancement against AIDS and Malnutrition) Programme of the Community of Sant'Egidio. Briefly, DREAM is a holistic programme started in December 2001 for the care of people living with HIV and the prevention of mother-to-child transmission (http://dream. santegidio.org). To date, the DREAM programme is active in ten countries (Mozambique, Malawi, Tanzania, Kenya, Republic of Guinea, Guinea Bissau, Cameroon, Democratic Republic of Congo, Angola and Nigeria) in SSA, with a network of 42 health centres and 20 laboratories. Because of the hub infrastructure used for health centres and laboratories in every country, only those facilities with molecular biology laboratories perform the HIV viral load testing. The possibility of using DBS to deliver samples for HIV viral load may therefore increase the accessibility to the test in rural areas and small settings. In this study, results were analysed according to different thresholds of HIV-RNA in order to develop an HIV viral load testing procedure from DBS that could be implemented in SSA.

\section{Methods}

Paired plasma and DBS samples were collected from 119 patients in 20 centres referring to the DREAM Health Centre of Blantyre, Malawi, and from 158 patients in 23 centres referring to the Centro Para Criança, Maputo, Mozambique. The DREAM Programme has been approved by the National AIDS Commission of Malawi and by the Ministério de Saude - Direcção de Planificação e Cooperação of Mozambique. 
Eight hundred microlitres of plasma were used to extract nucleic acids with the Abbott automatic extractor, Sample Preparation System (m2000SP). The nucleic acids extracted by m2000SP were used for real-time amplification with the Abbott m2000RT using the $0.6 \mathrm{~mL}$ HIV-RNA protocol. DBS ( $50 \mu \mathrm{L}$ of peripheral blood per spot) were air-dried overnight and stored in a plastic bag with desiccant at room temperature. DBS eluate was obtained by incubating two of five spots for each sample with $1.7 \mathrm{~mL}$ of Abbot Bulk lysis buffer for 15 minutes at room temperature with constant mixing. The eluate was transferred to a test tube and extracted using the m2000SP automatic extractor with the $1.0 \mathrm{~mL}$ HIV-1 RNA DBS Exp V04 protocol. The extracted material was then quantitated as the plasma samples with the real-time PCR Abbott m2000RT using the corresponding $1 \mathrm{~mL}$ DBS protocol, according to the manufacturer's instructions.

All HIV-RNA values were $\log _{10}$ transformed before analysis. Results reported as not detected and below detection limit $(<40$ copies $/ \mathrm{mL})$ were analysed considering a value of $1.6 \log _{10}$ HIV-RNA per mL. Results were expressed as means and standard deviations (SDs). Viral load measured in DBS and paired plasma samples were compared by the Pearson correlation analysis. The Bland-Altman method ${ }^{[8]}$ was used for analysis of concordance between the results obtained from plasma and those obtained from DBS. Statistical analysis was done using SPSS version 22 (IBM, USA).

\section{Results}

A total of 277 paired plasma and DBS samples were analysed. Samples were divided according to the HIV-RNA level in plasma: $\leq 2 \log _{10}$ ( $n=109), 2 \log _{10}$ (range $2.11-2.99 ; n=30$ ), $3 \log _{10}$ (range 3.10 - 3.99; $n=81$ ), $4 \log _{10}$ (range $4.04-4.96 ; n=29$ ) and $\geq 5 \log _{10}$ (range 5.03 6.84; $n=28$ ). Samples were analysed after a mean time of 1 week after collection and storage (at room temperature).

Agreement between detectable and undetectable viral load for plasma and DBS was seen in $83.0 \%$ of cases. In $16.6 \%$ of cases $(n=46)$, HIV-RNA was not detectable in DBS while measurable levels were present in plasma (between 1.76 and 3.83 logs; mean 2.48). One DBS gave a result of $3.54 \log _{10}$ while the corresponding plasma level was $<1.6 \log _{10}$. In all other cases of undetectable viral load in plasma ( $n=99$ ), HIV-RNA was not detectable in DBS. The lowest HIV-RNA level detected by DBS was 616 copies $/ \mathrm{mL}$.

Using the 40 copies $/ \mathrm{mL}$ threshold, the test had relatively good sensitivity (74\%), high specificity (99\%) and a high positive predictive value (99.2\%) (Table 1). Negative predictive value was low (68.3\%) as an effect of the low rate of detection when plasma HIV-RNA was below $3 \log$ copies $/ \mathrm{mL}$.
Among samples with detectable HIV-RNA, the correlation between viral load values obtained from the paired 131 plasma and DBS samples was high (Pearson correlation coefficient 0.946 and $R^{2}=0.895$ ) (Fig. 1). The mean (SD) difference between the measured viral load in DBS and in plasma was $0.15(0.27) \log$ copies/mL. HIV-RNA levels obtained from DBS were higher than in plasma in $70.2 \%$ of the samples. However, this over-quantification was observed mainly in specimens with a plasma viral load of $<3000$ copies $/ \mathrm{mL}$ (mean (SD) difference $0.38(0.19)$ ), while the difference for samples with a plasma viral load of $>3000$ copies $/ \mathrm{mL}$ was negligible $(0.09(0.26))$. Overall, viral load values between DBS and plasma differed by $<0.5 \log$ unit in $90.1 \%$ of cases and by $<1 \log$ unit in $100 \%$.

Agreement between the two methods was calculated by the BlandAltman method, ${ }^{\left[{ }^{8]}\right.}$ in which the differences between individual viral load results from plasma and DBS are plotted against the mean of the two results. In Fig. 2 it can be seen that the data are well scattered over the mean along the overall range of viral load examined and that all

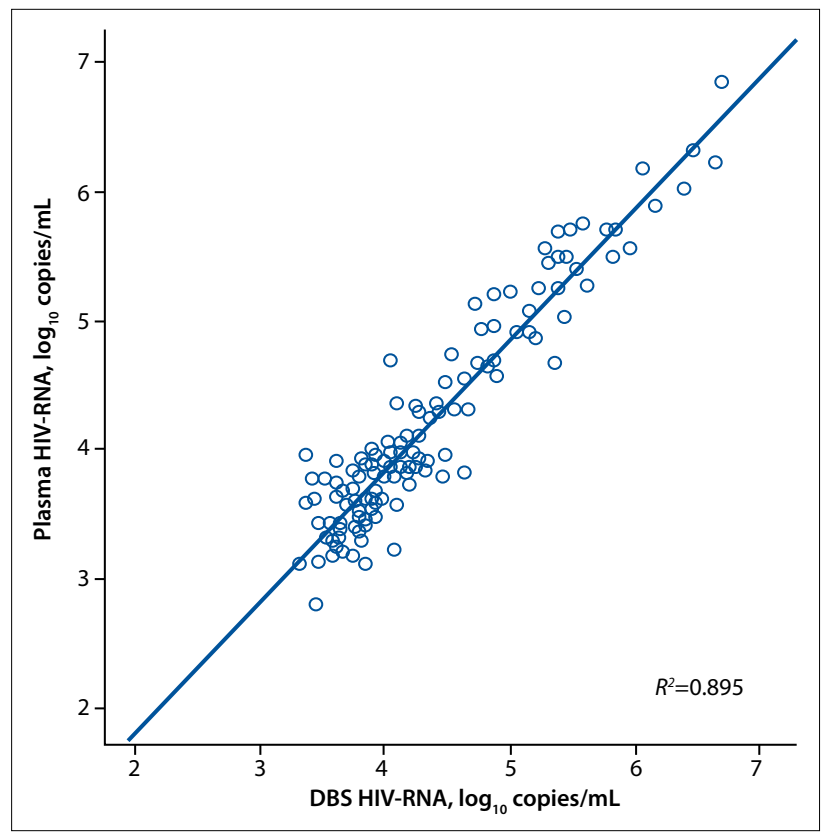

Fig. 1. Comparison of viral load in DBS and corresponding values in plasma only for samples with detectable viral load.

Table 1. Sensitivity, specificity, positive and negative predictive values (95\% confidence intervals) of DBS according to the limit of detection of the assay (A) and the threshold of 1000 copies $/ \mathrm{mL}(B)$

\begin{tabular}{|c|c|c|c|c|c|}
\hline \multicolumn{6}{|c|}{ Plasma } \\
\hline A & & $\geq 40$ copies $/ \mathrm{mL}$ & $<40$ copies $/ \mathrm{mL}$ & Total & \\
\hline \multirow[t]{5}{*}{ DBS } & $\geq 40$ copies $/ \mathrm{mL}$ & 131 & 1 & 132 & Sensitivity $74 \%(66.9-80.3)$ \\
\hline & $<40$ copies $/ \mathrm{mL}$ & 46 & 99 & 145 & Specificity $99 \%(94.5$ - 99.8) \\
\hline & Total & 177 & 100 & 277 & PPV 99.2\% (95.8 - 99.9) \\
\hline & & & & & NPV $68.3 \%(60.0-75.8)$ \\
\hline & & & Plasma & & \\
\hline B & & $\geq 1000$ copies $/ \mathrm{mL}$ & $<1000$ copies $/ \mathrm{mL}$ & Total & \\
\hline \multirow[t]{4}{*}{$\overline{\mathrm{DBS}}$} & $\geq 1000$ copies $/ \mathrm{mL}$ & 130 & 2 & 132 & Sensitivity $94.2 \%(88.9-97.5)$ \\
\hline & $<1000$ copies $/ \mathrm{mL}$ & 8 & 137 & 145 & Specificity $98.6 \%(94.9-99.8)$ \\
\hline & Total & 138 & 139 & 277 & PPV 98.5\% (94.6 - 99.8) \\
\hline & & & & & NPV 94.5\% (89.4 - 97.6) \\
\hline
\end{tabular}




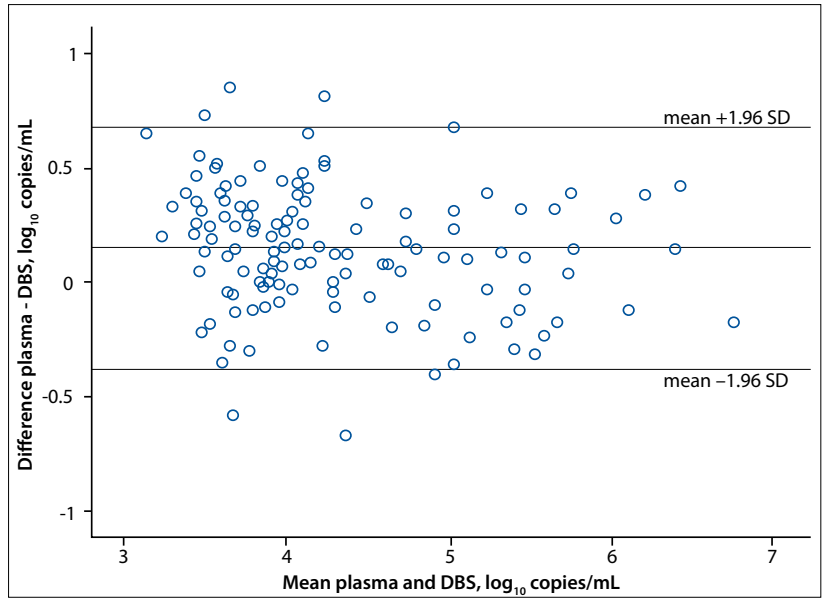

Fig. 2. Bland-Altman analysis of agreement between plasma and DBS. The horizontal lines represent the mean difference and 1.96 SDs.

but five samples were within the $1.96 \mathrm{SD}$ limits, underlining the good agreement between the two methods.

The detection rate with DBS was $90.1 \%$ when plasma levels were $>1000$ copies $/ \mathrm{mL}$. Categorising the samples according to this 1000 copies/mL threshold, of the 138 samples with a viral load of $>1000$ copies $/ \mathrm{mL}, 8(5.8 \%)$ had a DBS viral load of $<1000$ copies $/ \mathrm{ml}$ and therefore would have been missed if the virological failure threshold had been set at 1000 copies $/ \mathrm{mL}$. Of the 139 samples with a plasma viral load of $<1000$ copies/mL, only $2(1.4 \%)$ had an HIVRNA in DBS of $>1000$ copies $/ \mathrm{ml}$ and could represent false virological failure detection. With the threshold of 1000 copies $/ \mathrm{mL}$, sensitivity was $94.2 \%$, specificity $98.6 \%$ and positive and negative predictive values $98.5 \%$ and $94.5 \%$, respectively (Table 1 ). The agreement between DBS and plasma HIV-RNA quantification at the 1000 copies $/ \mathrm{mL}$ threshold was high, as demonstrated by the Kappa coefficient value of 0.928 (standard error 0.022, $p<0.001$ ).

\section{Discussion}

Several reports have been published on the use of DBS for viral load quantification using different methodologies, including the Abbott assay. ${ }^{[9]}$ In the great majority of cases the results were comparable with those obtained from plasma. However, the use of DBS collected and prepared in real-world conditions for viral load monitoring with the automated Abbott assay has been reported in only a few studies. ${ }^{[10-12]}$ Our data, obtained from DBS collected and processed by local personnel in Malawi and Mozambique, showed that DBS gave comparable results in respect of the reference method in the range from 3 to 7 logs copies/ $\mathrm{mL}$. However, the detection rate below the threshold of 1000 copies $/ \mathrm{mL}$ was lower, as reported in other articles, ${ }^{[2,13]}$ although the lowest plasma RNA value at which DBS RNA was detectable was 616 copies $/ \mathrm{mL}$ in line with the threshold of 550 copies/mL previously reported for this assay. ${ }^{[5]}$ The modest over-quantification that we observed with DBS can be ascribed to the presence of HIV-DNA and intracellular RNA and seems to have a greater impact on samples with a low viral load (in our study $<3000$ copies $/ \mathrm{mL}$ ), as previously reported. ${ }^{[4,11,14]}$

The 2013 HIV treatment guidelines of the World Health Organization $^{[15]}$ indicate a threshold of 1000 copies/mL of HIV-RNA to define virological failure using plasma, and suggest use of a higher, although undefined, threshold when using DBS. In previous studies performed with the Abbott system, sensitivity for the detection of virological failure at 1000 copies $/ \mathrm{mL}$ was between $75 \%$ and $100 \%$ and specificity was between $82 \%$ and $97 \% \cdot{ }^{[10,11]}$ In our study, very high positive and negative predictive values were observed when using a threshold of 1000 copies $/ \mathrm{mL}$, suggesting that even with the use of DBS the threshold of 1000 copies $/ \mathrm{mL}$ can provide reliable results, given the specificity of $98.6 \%$ at this threshold.

The last systematic review of the use of DBS for monitoring HIV viral load ${ }^{[9]}$ emphasised the need for a standardised approach for sampling, storing and processing DBS samples in order to establish whether DBS could reliably replace plasma specimens to identify virological failure. Our study suggests that DBS on filter paper, stored at room temperature and processed with an automated extractor, followed by real-time quantification of HIV-RNA, could represent a standard to be applied in resource-limited settings.

\section{Conclusion}

Our study demonstrated that HIV-1 quantification from DBS using a standardised commercial assay provides reliable identification of virological failure in SSA.

Acknowledgments. We acknowledge assistance of all the health workers of the health centres in Malawi and in Mozambique. The study was supported by the DREAM Programme, Community of Sant'Egidio, Rome, Italy.

\section{References}

1. Brambilla D, Jennings C, Aldrovrandi G, et al. Multicenter evaluation of use of dried blood and plasma spot specimens in quantitative assays for human immunodeficiency virus RNA: Measurement plasma spot specimens in quantitative assays for human immunodeficiency virus RNA: Measurement,
precision, and RNA stability. J Clin Microbiol 2003;41(5):1888-1893. [http://dx.doi.org/10.1128/ precision, and RNA stabill

2. Mbida AD, Sosso S, Flori P, et al. Measure of viral load by using the Abbott Real-Time HIV1 assay on dried blood and plasma spot specimens collected in 2 rural dispensaries in Cameroon J Acquir Immune Defic Syndr 2009;52(1):9-16. [http://dx.doi.org/10.1097/QAI.0b013e3181aeccbc]

3. Van Deursen P, Oosterlaken T, Andre P, et al. Measuring human immunodeficiency virus type 1 RNA load in dried blood spot specimens using NucliSENS EasyQ HIV-1 v2.0. J Clin Virol 2010;47(2):120125. [http://dx.doi.org/10.1016/j.jcv.2009.11.021]

4. Pirillo MF, Recordon-Pinson P, Andreotti M, Mancini MG, Amici R, Giuliano M. Quantification of HIV-RNA from dried blood spots using the Siemens VERSANT ${ }^{\circ}$ HIV-1 RNA (kPCR) assay. J Antimicrob Chemother 2011;66(12):2823-2826. [http://dx.doi.org/10.1093/jac/dkr383]

5. Arredondo M, Garrido C, Parkin N, et al. Comparison of HIV-1 RNA measurements obtained by using plasma and dried blood spots in the automated Abbott real-time viral load assay. J Clin Microbiol 2012;50(3):569-572. [http://dx.doi.org/10.1128/JCM.00418-11]

6. Johannessen A, Garrido C, Zahonero N, et al. Dried blood spots perform well in viral load monitoring Johannessen A, Garrido C, Zahonero N, et al. Dried blood spots perform well in viral load monitoring
of patients who receive antiretroviral treatment in rural Tanzania. Clin Infect Dis 2009;49(6):976-981. of patients who receive antiretrovir
[http://dx.doi.org/10.1086/605502]

Rottinghaus EK, Ugbena R, Diallo K, et al. Dried blood spot specimens are suitable alternative sample Rottinghaus EK, Ugbena R, Diallo K, et al. Dried blood spot specimens are suitable alternative sample
type for HIV-1 viral load measurement and drug resistance genotyping in patients receiving first-line antiretroviral therapy. Clin Infect Dis 2012;54(8):1187-1195. [http://dx.doi.org/10.1093/cid/cis015]

8. Bland JM, Altman DG. Statistical methods for assessing agreement between two methods of clinical measurement. Lancet 1986;1(8476):307-310.

Smit PW, Sollis KA, Fiscus S, et al. Systematic review of the use of dried blood spots for monitoring HIV viral load and for early infant diagnosis. PLoS One 2014;9(3):e86461. [http://dx.doi.org/10.1371/journal.pone.0086461]

10. Lofgren SM, Morrissey AB, Chevallier CC, et al. Evaluation of a dried blood spot HIV-1 RNA program for early infant diagnosis and viral load monitoring at rural and remote healthcare facilities. AIDS 2009;23(18):2459-2466. [http://dx.doi.org/10.1097/QAD.0b013e328331f702]

11. Monleau M, Aghokeng AF, Eymard-Duvernay S, et al. Field evaluation of dried blood spots for routine HIV-1 viral load and drug resistance monitoring in patients receiving antiretroviral therapy in Africa and Asia. J Clin Microbiol 2014;52(2):578-586. [http://dx.doi.org/ 10.1128/JCM.02860-13]

12. Rutstein SE, Kamwendo D, Lugali L, et al. Measures of viral load using Abbott RealTime HIV-1 Assay on venous and fingerstick dried blood spots from provider-collected specimens in Malawian district
ons on venous and fingerstick dried blood spots from provider-collected specimens in Mala
hospitals. J Clin Virol 2014;60(4):392-398. [http://dx.doi.org/10.1016/j.jcv.2014.05.005]

hospitals. J Clin Virol 2014;60(4):392-398. [http://dx.doi.org/10.1016/j.jcv.2014.05.005]
13. Marconi A, Balestrieri M, Comastri G, et al. Evaluation of the Abbott Real-Time HIV-1 quantitative 3. Marconi A, Balestrieri M, Comastri G, et al. Evaluation of the Abbott Real-Time HIV-1 quantitative
assay with dried blood spot specimens. Clin Microbiol Infect 2009;15(1):93-97. [http://dx.doi org/10.1111/j.1469-0691.2008.02116.x]

14. Vidya M, Saravanan S, Rifkin S, et al. Dried blood spots versus plasma for the quantitation of HIV1 RNA using a real-time PCR, m2000rt assay. J Virol Methods 2012;181(2):177-181. [http://dx.doi. org/10.1016/j.jviromet.2012.02.006]

15. World Health Organization. 2013. Consolidated guidelines on the use of antiretroviral drugs for treating and preventing HIV infection: Recommendations for a public health approach. http://www. who.int/hiv/pub/guidelines/arv2013/download/en/index.html (accessed 15 October 2015).

Accepted 5 October 2015 